\title{
A METHOD OF CONTROL FOR POSTOPERATIVE ADENOIDAL BLEEDING
}

\author{
C. G. McNeILL, M.D. ${ }^{*}$
}

ANTHONY has suggested an ingenious method of nasopharyngeal packing by using a Foley catheter of appropriate size and inflating the catheter after insertion. ${ }^{1,2}$ For the past five years, members of the Department of Anaesthesia of the Lion's Gate Hospital in North Vancouver have been achieving similar results by using endotracheal catheters. This method has the advantage of maintaining a nasopharyngeal airway while packing off the surrounding area.

Bleeding from the adenoid space after adenoidectomy can usually be controlled in the following manner. Select a cuffed endotracheal tube of the largest diameter which can be introduced through the child's nares. Lubricate the tube

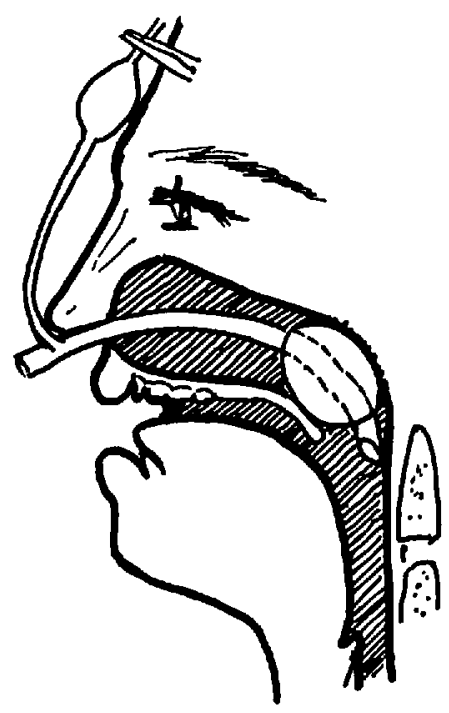

Figure 1

and insert it until the cuff approximates the adenoidal space. Inflate the cuffusually about 10 c.c. The tube will slide into place with the balloon secure in the fossa. (See Fig. 1.) Check the pharynx to make sure the balloon is in position and not in the pharynx. The tube should be visible beyond the uvula, and the soft palate not pushed too far down. Leave the tube in place for fifteen to twenty minutes. It can be reinserted if bleeding recommences. It may be left in overnight.

This method is less traumatic and less hazardous than packing. It can be accomplished in the postoperative period without anaesthetic and without too

- Department of Anaesthesia, Vancouver General Hospital. Formerly Chief of General Practice, Lion's Gate Hospital, North Vancouver. 
much struggling. It has the advantage of providing an airway. It gives ready access for suctioning the pharynx. It is a method worth trying when one is not sure whether the bleeding is from the tonsils or from the adenoids. It has been used in severe epistaxis in adults. The posterior nares is closed off with the balloon. Packing is inserted through the anterior nares and the entire space occluded.

The size of the endotracheal tube to be selected is determined by the size of the nostril of the child. The inflatable balloon provides the same haemostasis regardless of the size of the tube but the larger the tube the larger the nasopharyngeal airway provided. The balloon should be roughly spherical and the distal tip of the catheter no more than $15 \mathrm{~mm}$. in length to prevent it from irritating the pharynx.

The only difficulties that have been encountered have occurred when too large an amount of air has been inserted into the balloon. This results in the soft palate being pushed down uncomfortably onto the tongue. If this appears to be the case some air should be withdrawn and the airway reinspected. Because of his familiarity with endotracheal tubes it is recommended that the anaesthetist be the one to insert it, to blow up the cuff, and to check its position. If it is to be left in while the child awakes it should be taped firmly to the nose and cheek. The tell-tale balloon should have an easily removable clamp rather than being tied.

\section{CAse Report No. I}

The nurse in the postanaesthetic recovery room reported that a four-year-old girl who had just had an adenotonsillectomy was bleeding from the nostrils and had just vomited blood-stained gastric fluid. There had been no troublesome bleeding from the tonsil site and it was suspected that the bleeding was coming from the adenoidal area.

A number 3 endotracheal catheter with cuff was inserted by way of the larger nostril and the cuff was inflated in the adenoidal space. With 7 c.c. of air it fitted snugly in position. No further bleeding occurred and the tube was removed before discharge from the postanaesthetic recovery room.

\section{Case Report No. II}

Persisent bleeding occurred from the operative site in a six-year-old child. This was controlled in the tonsillar area by ligation of the bleeding points. In the adenoidal area bleeding was stopped by packing with a gauze sponge but recommenced when the sponge was withdrawn. A number 24 cuffed catheter was inserted by way of the larger nostril and the cuff was inflated with 8 c.c. of air. Inspection showed the tip of the catheter to be in the pharynx. Pressure of the cuff was continued for 10 minutes at which time the cuff was released. Bleeding recommenced so it was again inflated. The patient was sent to the postanaesthetic recovery room with the cuff in position. An oral airway was required. Air could be felt to be moving through both the oral and the nasal airway. The cuff was released in 20 minutes. There being no further bleeding, the tube was withdrawn. 
Case Report No. III

One week after an adenotonsillectomy, a five-year-old boy was readmitted to hospital because of haemorrhage from the operative site. He was pale with a $\mathrm{Hgb}$. of $7.1 \mathrm{gm}$., pulse 110 per minute, blood pressure 80/60. Blood was matched for transfusion and a general anaesthetic given. Both tonsillar areas were found to be dry and the bleeding to be coming from the adenoidal site.

A number 24 cuffed endotracheal tube was introduced by way of the left nostril until the tip protruded beyond the soft palate. The cuff was inflated with 8 c.c. of air until it fitted snugly in the adenoidal space. After ten minutes the cuff was released and bleeding recommenced. It was again blown up and the patient allowed to awaken. He was sent to the postanaesthetic recovery room and the tube was left in situ overnight. In the morning the cuff was again released. No further bleeding occurred so the tube was removed.

\section{SUMMARY}

A method is described of nasopharyngeal packing to control bleeding from the adenoidal site following adenotonsillectomy. The method involves the use of an endotracheal tube inserted in the nares, the cuff being inflated in the nasopharynx to provide pressure on the bleeding points. Three cases are reported in which this procedure was successfully employed.

\section{RÉSUMÉ}

On décrit une technique de paquetage naso-pharyngé destiné à controler le saignement dans la région adénoidienne après une adéno-amygdalectomie. II s'agit d'utiliser un tube endotrachéal introduit dans une narine, et d'insuffler le ballonnet dans le naso-pharynx pour produire une pression sur les points de saignement. On rapporte trois cas qui ont bénéficié de cette technique.

\section{REFERENCES}

1. Hill, Frederick T. Otolaryngology, vol. IV. Hagerstown: W. F. Prior (1960), p. 45.

2. GregG, Jorn B. Treating the Complications of Tonsillectomy-Adenoidectomy, GP. 26: 82 (1962). 